compared with treated patients $(12 \%$ versus $26 \%$, respectively; $\mathrm{p} \leq 0.01)$.

Conclusions Despite recent regulatory approval of antifibrotic therapies, many European patients with confirmed IPF do not receive approved antifibrotic treatment. Possible explanations may include: lack of MDT diagnosis; lack of referral to specialist centres; patients not meeting treatment thresholds; subjective perceptions of disease severity; reluctance to treat patients with 'stable' disease; variations in patient/physician awareness or knowledge of IPF; or lack of confidence in prescribing new treatments.

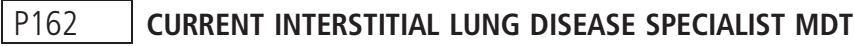 PROVISION ACROSS THE UK}

TA Mikolasch, H Garthwaite, J Porter. University College London, London, UK

10.1136/thoraxjnl-2016-209333.305

The advent of novel anti-fibrotic therapies and the introduction of specialist, commissioned Interstitial Lung Disease (ILD) centres, has led to an increased workload for Multidisciplinary Team (MDT) meetings. We set out to survey specialist UK centres to gain a better understanding of their organisational processes and associated challenges.

Methods Between August and December 2015 we conducted an online survey of all 23 NHS England commissioned ILD centres, plus 5 specialist ILD centres in Scotland, Wales and Northern Island. The survey was sent to the clinical lead of each centre. A total of 20 questions assessed the workforce composition and frequency of meetings. Their workload was also evaluated and we asked them to identify areas that required improvement.

Results 26 out of 28 centres responded.

MDTs are coordinated by the ILD lead consultant $(57 \%)$ or a medical secretary (26\%), with only $17 \%$ directed by a MDT coordinator.

Peripheral hospitals participate in MDTs in 78\% of centres; in person, via video-link or paper referrals; however, the majority of discussed patients are reviewed at the specialist centre.

MDTs are typically held weekly, lasting 1 to 2 hours, with 10 to 20 patients discussed. $26 \%$ of MDTs discuss all new referrals, $87 \%$ discuss all patients considered for anti-fibrotic therapy, whilst only $22 \%$ discuss all patients considered for immunosuppressive therapy (aside from oral steroids).

All respondents agreed that the available MDT time was insufficient. The most common reasons were cited as; lack of dedicated MDT funding (83\%), lack of sufficient respiratory radiologist consultant time (78\%) and lack of dedicated administrative support (61\%).

In $96 \%$ of cases there is no local tariff in place to fund MDT discussion and all respondents agreed that a dedicated tariff would improve MDT provision.

92\% of centres enrol MDT patients into clinical trials.
Conclusion Specialist ILD MDTs are able to concentrate a high level of expertise and allow patients access to vital clinical trials. They are, however, under considerable strain due to lack of funding and administrative support. A dedicated funding stream for this specialist service would be beneficial.

\section{P163 SURGICAL LUNG BIOPSY IN THE DIAGNOSIS OF INTERSTITIAL LUNG DISEASE- A SYSTEMATIC LITERATURE REVIEW}

${ }^{1}$ TA Mikolasch, ${ }^{2} \mathrm{~A}$ Marshall, ${ }^{1} \mathrm{~A}$ Salam, ${ }^{1} \mathrm{JC}$ Porter. ${ }^{1}$ University College London, London, UK; ${ }^{2}$ University of Edinburgh, Edinburgh, UK

10.1136/thoraxjnl-2016-209333.306

Methods We performed a systematic literature review based on the PRISMA guidelines following a pre-specified protocol. Pubmed and Embase databases were searched for studies reporting the use of Surgical Lung Biopsies (SLB) in the diagnosis of adults with Interstitial Lung Disease (ILD). Randomised controlled trials, case control studies and case series with more than 20 subjects were included, restricted to papers published from 2000 till September 2015 taking into consideration changes in surgical techniques and diagnostic criteria. All relevant abstracts were assessed by two independent reviewers utilising EPPI reviewer 4, an online software tool for research synthesis. Full papers were obtained for those deemed potentially eligible, and two reviewers agreed the final set of review papers. Primary outcomes were 90 day mortality and complications while secondary were diagnostic yield, mean length of stay and change of treatment following biopsy.

Results (see Figure 1). 24 studies were included reporting on the use of SLB in 2600 patients. The overall quality of the reports was moderate to poor with mainly retrospective case series available. Mean mortality was $4.9 \%$ (CI 90\% - $0.04-0.14$ ) with a wide range of $0-22.4 \%$. Complication rates were reported in 19 of the studies. Mean overall complication rate was $19.4 \%$ (CI $-0.05-0.48$ ) with a range from $7.1 \%$ to $65.7 \%$. Mean length of stay adjusted for patient numbers was 5.4 days and diagnostic yield for definite pathological diagnosis was $89 \%$. Eight studies recorded treatment change following SLB in a total of 588 patients out of 869 . Mean percentage of patients in whom treatments was changed on the basis of the SLB result was 60\% (CI 90\% 0.35-0.87).

Conclusions High-quality data on the outcomes of SLB in ILD diagnosis are sparse. Comparison between different studies is difficult due to heterogeneous patient populations (e.g. acute vs elective cases) and differences in outcome reporting. Nonetheless, the overall mortality and morbidity rates are similar to a recent analysis of a US national database. SLB in ILD remains a useful diagnostic tool but carries significant mortality and morbidity. More prospective data and evaluation of surgical risk stratification is required. 


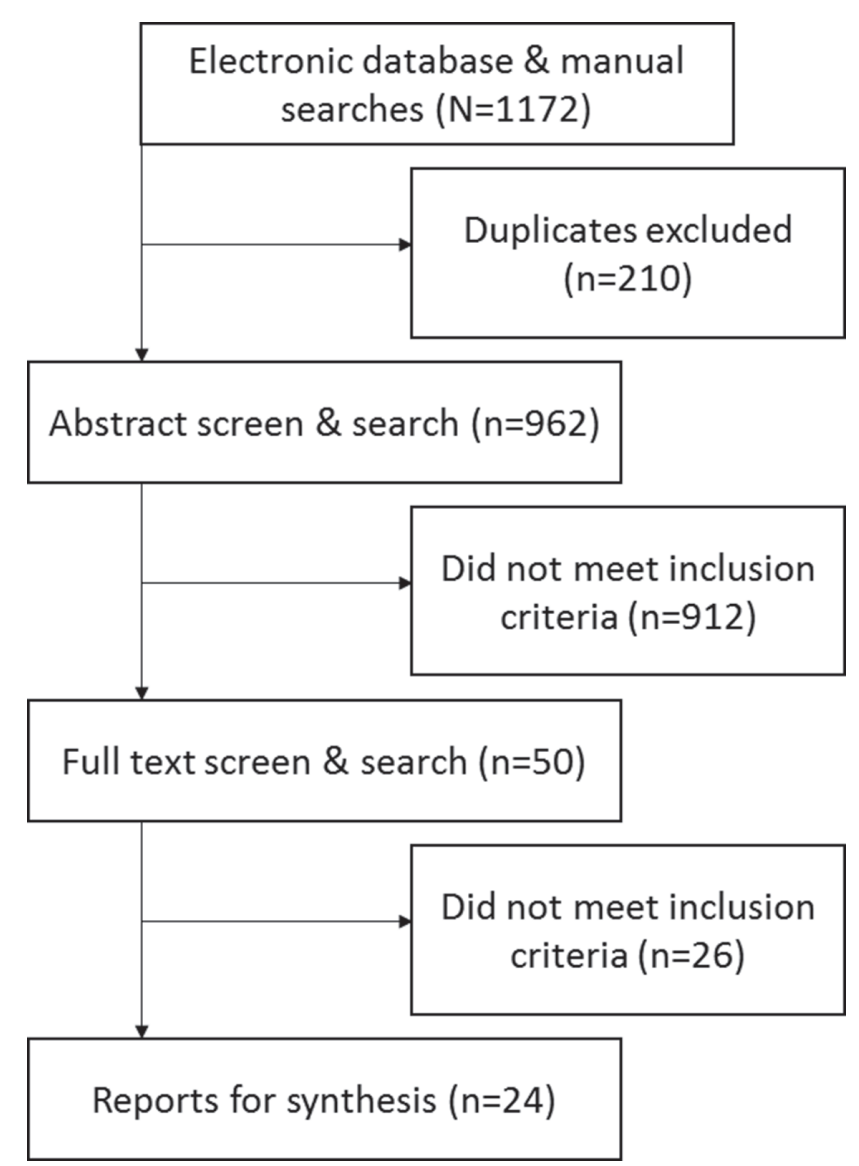

Abstract P163 Figure 1 PRISMA flow diagram of evidence synthesis

\section{P164 CHANGING PATTERNS OF THE USE OF LUNG BIOPSY IN INTERSTITIAL LUNG DISEASE}

L Brockbank, E Hilal, J Holemans, J Greenwood, M Walshaw, K Mohan. Liverpool Heart and Chest Hospital, Liverpool, UK

\subsection{6/thoraxjnl-2016-209333.307}

Introduction Radiological and international guidelines have improved the diagnosis of interstitial lung disease (ILD) subtype in the absence of a surgical lung biopsy (SBX). However, it may still be needed since up to $38 \%$ of cases ${ }^{1}$ cannot be diagnosed on clinical and radiological grounds alone, and new antifibrotic therapies require more diagnostic certainty for idiopathic pulmonary fibrosis (IPF). We wished to ascertain whether SBx rates and diagnostic outcomes had changed at our regional thoracic centre. Methods We looked at 104 consecutive patients undergoing SBx between May 2014 to April 2016, and compared their mode of referral and outcome with a previous study (210 cases) in the same centre conducted between 2001 and 2008.

Results There was no evidence of multidisciplinary team (MDT) input prior to SBx in 31 cases $(30 \%)$, but 18 (17\%) were discussed at an ILD MDT and 55 (53\%) in local radiology meetings. For SBx outcome see Table. Prior diagnosis was uncertain in $28 \%$ of ILD MDT cases and $27 \%$ of local radiology meeting cases, whereas SBx confirmed the suspected diagnosis in 22\% of ILD MDT cases but only $9 \%$ of radiology meeting cases.
Conclusion Overall, there appears to be increase in the ILD cases referred for SBx. Despite the small proportion of cases discussed at the ILD MDT prior to SBx, there appears to be a trend in the reduction of UIP/NSIP and significant increase in HSP, RBILD and DIP cases. Histological diagnosis remains important in ILD, and the use of other techniques with lower complication rates (e. g. transbronchial cryobiopsy) needs to be established.

\begin{tabular}{|c|c|c|c|}
\hline Diagnosis & $\begin{array}{l}\text { Percentage of cases } \\
2001-2008(n=210)\end{array}$ & $\begin{array}{l}\text { Percentage of cases } \\
2014-2016(n=104)\end{array}$ & $P=$ \\
\hline $\begin{array}{l}\text { Usual interstitial } \\
\text { pneumonia (UIP) }\end{array}$ & $37 \%$ & $29 \%$ & 0.17 \\
\hline $\begin{array}{l}\text { Non-specific interstitial } \\
\text { pneumonia (NSIP) }\end{array}$ & $12 \%$ & $7 \%$ & 0.17 \\
\hline Organising pneumonia & $10 \%$ & $3 \%$ & 0.025 \\
\hline Sarcoidosis & $8 \%$ & $10 \%$ & 0.53 \\
\hline $\begin{array}{l}\text { Smoking related (RB-ILD, } \\
\text { DIP) }\end{array}$ & $9 \%$ & $23 \%$ & 0.0014 \\
\hline $\begin{array}{l}\text { Hypersensitivity } \\
\text { pneumonitis }\end{array}$ & $4 \%$ & $12 \%$ & 0.0072 \\
\hline $\begin{array}{l}\text { Vasculitis/Connective } \\
\text { tissue disease }\end{array}$ & $2 \%$ & $1 \%$ & $>0.99$ \\
\hline Other & $18 \%$ & $15 \%$ & 0.43 \\
\hline
\end{tabular}

\section{REFERENCE}

1 Raghu G, Mageto YN, Lockhart D, et al. The accuracy of the clinical diagnosis of new-onset idiopathic pulmonary fibrosis and other interstitial lung disease. A prospective study. Chest 1999;116:1168-1174.

\section{P165 SURGICAL LUNG BIOPSY IN THE DIAGNOSIS OF INTERSTITIAL LUNG DISEASE - WHERE ARE WE NOW?}

L Brockbank, E Hilal, L Johns, M Walshaw, K Mohan. Liverpool Heart and Chest Hospital, Liverpool, UK

\subsection{6/thoraxjnl-2016-209333.308}

Introduction With the advent of multidisciplinary team (MDT) working and new therapies in interstitial lung disease (ILD), diagnostic accuracy is increasingly important, and international guidelines ${ }^{1}$ have reaffirmed the importance of surgical lung biopsy (SBx) where necessary. However, SBx has associated risks: to assess this further we looked at the diagnostic yield and complication rate of SBx carried out at our regional thoracic centre for patients with ILD.

Methods We looked at all 104 SBxs carried out for ILD over 24 months between 2014-16, collecting data on the nature of the procedure, number of lobes sampled, complications encountered and mortality, and also whether the cases had been discussed at a regional ILD or local radiology MDT meeting prior to SBx.

Results Seventy cases (67\%) had been discussed prior to SBx (18 at an ILD MDT). Overall, mean age was 56 years, mean FEV1 $79 \%$ predicted, FVC $84 \%$ predicted, RV $79 \%$ predicted, TLC $77 \%$ predicted, TLCO $56 \%$ predicted, and KCO 77\% predicted. All but 3 procedures were carried out by VAT: the median number of lobes sampled was 2 (>1 lobe in $86 \%$ ), and diagnostic specimens were obtained in 97\% (UIP 29\%, RB-ILD and DIP $23 \%$, HSP $12 \%$, Sarcoid 10\%, NSIP 7\%, others 19\%). For complications see Table. The mean length of stay was 5.2 days (range 1-44): in-hospital mortality and 30-day mortality were $1 \%$ and $3 \%$ respectively. 